\title{
Comparison of the Sentinel-1B Synthetic Aperture Radar With Airborne Microwave Sensors in an Extra-Tropical Cyclone
}

Sapp Joseph W. ${ }^{1,2,{ }^{*}}$, Mouche Alexis ${ }^{3}$, Jelenak Zorana ${ }^{2,4}$, Chang Paul S. ${ }^{2}$, Frasier Stephen J. 5

1 Global Sci \& Technol GST Inc, College Pk, MD 20740 USA.

2 NOAA, Natl Environm Satellite Data \& Informat Serv NESD, College Pk, MD 20740 USA.

3 IFREMER, Lab Oceanog Phys \& Spatiale, F-29280 Plouzane, France.

4 Univ Corp Atmospheric Res UCAR, Boulder, CO 80307 USA.

5 Univ Massachusetts, Microwave Remote Sensing Lab, Amherst, MA 01003 USA.

*Corresponding author : Joseph W. Sapp, email address : joe.sapp@noaa.gov

\begin{abstract}
:
In Winter 2017, the University of Massachusetts Amherst's Imaging Wind and Rain Airborne Profiler (IWRAP) was flown on a National Oceanic and Atmospheric Administration (NOAA) WP-3D Hurricane Hunter aircraft under the direction of scientists from Center for Satellite Applications and Research (STAR) at NOAA/National Environmental Satellite, Data, and Information Service (NESDIS) over the North Atlantic ocean out of Shannon, Ireland. IWRAP is a dual-frequency, conically scanning, profiling Doppler radar initially developed by Microwave Remote Sensing Laboratory (MIRSL) at the University of Massachusetts Amherst that is routinely installed on the NOAA WP-3D research aircraft. The flight on February 6, 2017, targeted a region of high winds (greater than $30 \mathrm{~m} / \mathrm{s}$ ) that was also observed by the Sentinel-1B satellite's synthetic aperture radar. Sentinel-1B was configured to observe in extended wide swath mode in both VV- and VH-polarizations, whereas the IWRAP C-band radar was configured to measure all of VV-, VH-, and $\mathrm{HH}$-polarizations. IWRAP and Sentinel-1B VV and VH normalized radar cross section (NRCS) at the same Earthincidence angle along the flight path match reasonably well during the entire flight, but some additional trends between aircraft and satellite can be observed. IWRAP VVpolarized NRCS generally match the CMOD5.h geophysical model function (GMF), suggesting errors in the Sentinel-1B processing chain.
\end{abstract}

Keywords : Satellites, Aircraft, Spaceborne radar, Synthetic aperture radar, Sea measurements, Wind speed, C-band, cross polarization, normalized radar cross section (NRCS), ocean winds, scatterometry 


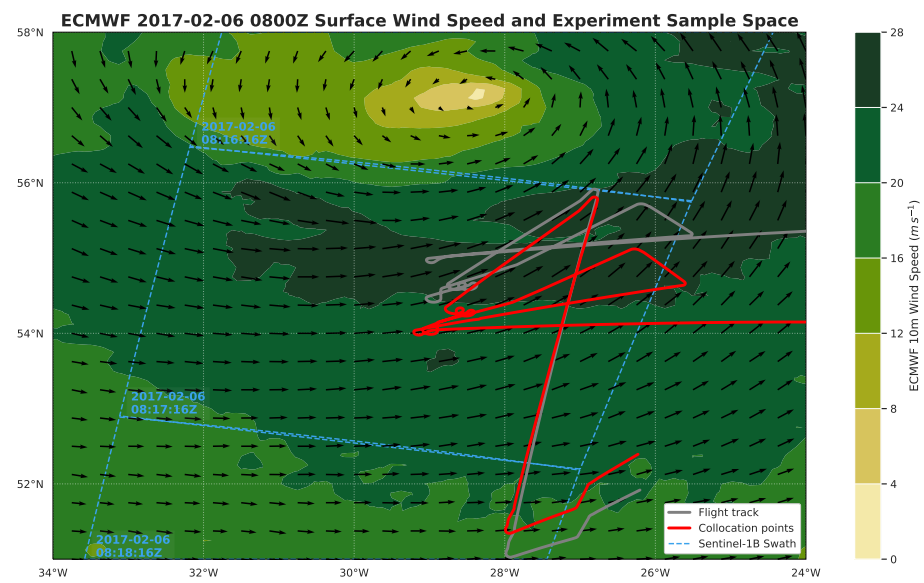

\section{InTroduction ANd EXPERIMENT Description}

$\mathbf{S}$

sea-surface backscatter are becoming popular for retrieving wind speeds from tropical cyclones because of their high spatial

Manuscript received May 8, 2019; revised November 15, 2019; accepted December 28, 2019. This work was supported by the National Oceanic and Atmospheric Administration Ocean Winds program, European Space Agency SMOS+STORM evolution through its Support to Science Element Program under Contract CCN2 4000105171/12/I-BG, and European Space Agency Sentinel1 for Science Ocean Study through its Support to Scientific Exploitation of Operational Missions Element Program under Contract S1-4SCI-16-0002. (Corresponding author: Joseph W. Sapp.)

Joseph W. Sapp is with Global Science \& Technology (GST), Inc., Greenbelt, MD 20740 USA (e-mail: joe.sapp@noaa.gov).

Alexis A. Mouche is with Laboratoire d'Océanographie Physique, Spatiale, IFREMER, 29280 Plouzané, France.

Zorana Jelenak is with University Corporation for Atmospheric Research (UCAR), Boulder, CO, 80307, USA.

Paul S. Chang is with the National Oceanic and Atmospheric Administration (NOAA)/National Environmental Satellite, Data, and Information Service (NESDIS) Center for Satellite Applications and Research (STAR), College Park, MD 20740 USA.

Stephen J. Frasier is with the Microwave Remote Sensing Laboratory, University of Massachusetts Amherst, Amherst, MA 01003 USA.

Color versions of one or more of the figures in this paper are available online at http://ieeexplore.ieee.org.

Digital Object Identifier 10.1109/TGRS.2020.2966332

0196-2892 @ 2020 IEEE. Personal use is permitted, but republication/redistribution requires IEEE permission.

See https://www.ieee.org/publications/rights/index.html for more information.
Figure 1. Flight track of the NOAA WP-3D (gray) and the collocation points with Sentinel-1B (red) on top of ECMWF wind contours and vectors. Sentinel$1 \mathrm{~B}$ acquisition areas are shown as large trapezoids with the acquisition time, approximately $0817 \mathrm{Z}$ for most of the collocated points, in the bottom left corner. The Sentinel-1B nadir is to the right of the acquisition swath in the image, so the Earth-incidence angles increase from right to left.

resolution and cross-polarization capabilities [1], despite the inherent challenges in retrieving wind direction. It is also difficult to calibrate and validate these instruments for use at all antenna elevation angles and high wind speeds because of their sparse data acquisition, but some satellite intercomparison is possible [2]. In particular, the very low backscattered crosspolarized signal imposes a severe constraint on the satellite noise estimate and correction. The Ocean Surface Winds Team from the Center for Satellite Applications and Research (STAR) at the National Oceanic and Atmospheric Administration (NOAA) National Environmental Satellite, Data, and Information Service (NESDIS) routinely performs airborne flight experiment campaigns for the purpose of collecting remotely-sensed and in-situ data in order to calibrate, validate, and improve the understanding of measurements from spaceborne sensors. The primary purpose of this manuscript is to evaluate the performance of the cross-polarized measurements from the Sentinel-1B SAR across subswaths in a rain-free high-wind environment. This has implications for satellite intercomparison with Sentinel-1B, wind speed retrievals from Sentinel-1B, and development of future cross-polarized geophysical model functions. We also identify areas of improvement for the calibration of Sentinel-1B. 
On February 6, 2017, the authors directed a flight of a NOAA WP-3D over an extratropical cyclone in the North Atlantic while the Sentinel-1B SAR sampled the same region. Wind speeds were in excess of $30 \mathrm{~m} \mathrm{~s}^{-1}$, providing an opportunity to compare normalized radar cross-section (NRCS) from spaceborne SAR with aircraft measurements over a large field of near-hurricaneforce winds. An additional benefit to this storm in particular, and extratropical cyclones in general, is that the environment was cold and dry. There was no liquid precipitation under the aircraft, according to onboard instrumentation. These conditions greatly reduce the likelihood of enhanced surface roughness due to rain and create a stable environment for comparing the SAR with airborne sensors over a few hours. Figure 1 shows the flight track over the European Centre for Medium-Range Weather Forecasts (ECMWF) wind vectors from 0800Z. Sentinel-1B acquisition areas are shown as large trapezoids.

The Sentinel-1 mission is a constellation of two polar-orbiting satellites, each carrying a C-band $(5.405 \mathrm{GHz})$ synthetic aperture radar, and is operated by the European Space Agency for the operational needs of the Copernicus program. The Sentinel$1 \mathrm{C}$-band SAR supports operation in single polarization $(\mathrm{HH}$ or $\mathrm{VV}$ ) and dual polarization $(\mathrm{HH}+\mathrm{HV}$ or $\mathrm{VV}+\mathrm{VH})$ and have four exclusive acquisition modes. During the NOAA flight experiment on 6 Feb 2017 it was operated in extended wide swath (EW) mode, which is $400 \mathrm{~km}$ wide and observes the Earth at Earth-incidence angles (EIAs) from $17^{\circ}$ to $45^{\circ}$. EW mode captures five sub-swaths using Terrain Observation by Progressive Scans SAR (TOPSAR). With this technique, in addition to steering the beam in range as in ScanSAR, the beam is also electronically steered from backward to forward in the azimuth direction for each burst [3]. The data presented here were from the two subswaths that correspond to the lowest EIA (i.e., near-range-approximately $16^{\circ}$ to $35^{\circ}$ incidence, depending on orbit altitude). We performed noise correction and averaging on the Sentinel-1B data. The final pixel spacing is $1 \mathrm{~km}$ but the resolution is $3 \mathrm{~km}$.

The NOAA WP-3D carried the Imaging Wind and Rain Airborne Profiler (IWRAP) [4], [5], a C- and Ku-band scatterometer system designed and developed by the University of Massachusetts Amherst, and the Stepped Frequency Microwave Radiometer (SFMR), a C-band microwave radiometer. Though SFMR was developed for use in tropical environments, coldweather corrections have been applied so that they match GPS dropwindsondes better [6].

IWRAP is a Doppler radar that was originally designed to support a conical scan at multiple incidence angles and polarization configurations. An internal calibration loop allows for tracking of receiver gain and transmit power during the flight. Since winter 2015, the C-band radar has been configured for a fan-beam antenna on loan from ESA [7], [8]. This antenna has a better cross-polarization isolation (greater than $40 \mathrm{~dB}$ isolation across the main lobe) than the C-band IWRAP pencil-beam phased array, allowing for measurements of cross-polarized NRCS at low wind speeds. It is statically mounted and, since summer of 2015, pointing $25^{\circ}$ off nadir and towards the right of the aircraft. The IWRAP C-band radar transmits a pulse-compressed waveform (i.e., chirp) with a $4 \mathrm{MHz}$ bandwidth at the antenna's design frequency of $5.3 \mathrm{GHz}$. The ESA antenna on IWRAP has an azimuthal (along the direction of the aircraft fuselage) beamwidth of approximately $5^{\circ}$ across the main lobe. This equates to approximately 80 to $125 \mathrm{~m}$ on the surface for $20^{\circ}$ to $30^{\circ} \mathrm{EIA}-$ the range used in this manuscript - but the spatial resolution was coarsened to $1 \mathrm{~km}$ in the direction of the aircraft flight track to reduce noise levels and better match the spatial resolution of the Sentinel-1B SAR. IWRAP has a range gate spacing of $30 \mathrm{~m}$, but the cross-track resolution (along the direction of the aircraft wings) on the ground is approximately 90 to $60 \mathrm{~m}$ across the same EIA range. So even though IWRAP has a comparable resolution along the flight track, it has a much smaller crosstrack resolution. Both $\mathrm{V}$ and $\mathrm{H}$ polarizations are available on transmit and receive and, for this experiment, IWRAP measured NRCS at VV, HH, and VH. With the exception of the C-band antenna mount angle, the configuration is the same as in [5]. While the $\mathrm{Ku}$-band radar was operational at two incidence angles for $\mathrm{VV}$ and $\mathrm{HH}$, the $\mathrm{C}$-band radar is the radar of interest in this manuscript. The C-band fan-beam antenna allows selection of NRCS from approximately $15^{\circ}$ to $45^{\circ}$ EIA at level flight and the typical altitude of 2 to $3 \mathrm{~km}$.

The SFMR has a $3 \mathrm{~dB}$ beamwidth of $20^{\circ}$ to $28^{\circ}$ and it takes approximately $4 \mathrm{~s}$ to step through all frequencies [9]. For the flight on 2017 Feb 6, this beamwidth equated to a cross-track swath of 750 to $1050 \mathrm{~m}$, which is close to the spatial scale of the Sentinel-1B measurements used and the alongtrack averaging scale of IWRAP. During the integration time, the aircraft traveled $0.5 \mathrm{~km}$ on average. Using the same beamwidth values as the cross-track estimates, this yields an alongtrack averaging distance of 1250 to $1550 \mathrm{~m}$. These small differences and variations in spatial scale are not expected to change the analysis since surface wind speeds are only used to adjust Sentinel-1B VV NRCS and to check for biases in IWRAP. As will be described in the next section, adjustments to correct for different azimuth look angles are made only to the Sentinel-1B VV NRCS. All VH conclusions are independent of wind speed.

All sensors have similar spatial resolutions, with the exception of the IWRAP cross-track resolution. This will result in noisier IWRAP data, but otherwise do not affect the analysis.

The remainder of this paper is structured to show an elevation angle-dependent NRCS difference between Sentinel-1B and IWRAP, especially as a function of subswath. Elevation angle was chosen for most of the discussion because the data suggest a radar calibration problem rather than a geophysical phenomena. This is more naturally expressed for a satellite as a function of elevation angle.

Section II describes how the sensors were aligned in time and space to measure the same ocean-surface phenomena; Section III details the IWRAP calibration procedures; and Section IV presents and interprets intercomparison results.

\section{Collocation Methodology}

Collocation of airborne sensors with spaceborne sensors in extreme environments is challenging due to the speed difference of the two platforms and the movement of the storm. While it took minutes for Sentinel-1B to image the area, the WP-3D took hours, during which the location, direction, and magnitudes of the winds initially sampled by the SAR changed. In order 


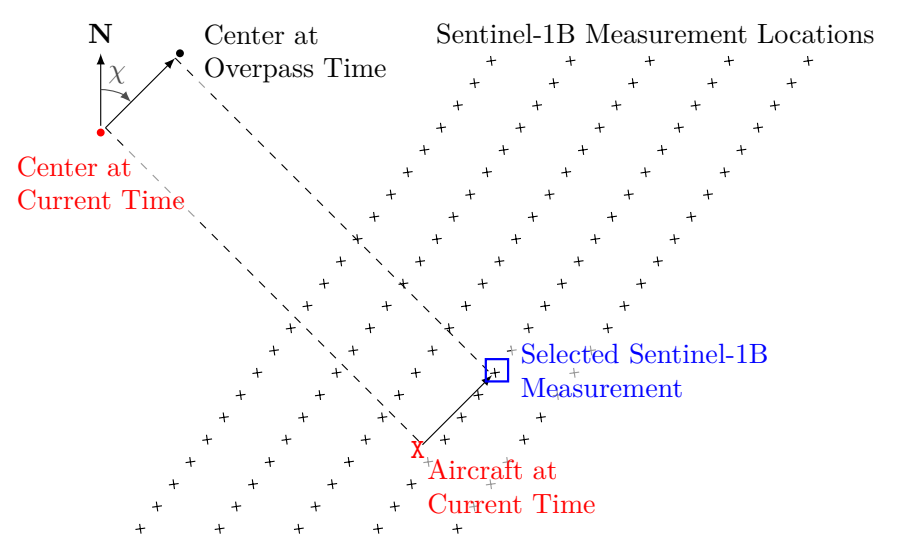

Figure 2. A schematic diagram showing how the Sentinel-1B measurement location is selected. The aircraft location is translated in the same bearing $\chi$ and distance (in kilometers) that the storm center is from the current time to the satellite overpass time. The nearest Sentinel-1B measurement to the resulting location is selected for collocation. All IWRAP alongtrack cells are passed through this procedure.

to account for the storm displacement, we used storm-relative positions to collocate the two instruments.

Aircraft locations and times were first grouped into $1 \mathrm{~km}$ alongtrack cells. Then we estimated the storm center based on the minimum wind speed location from spatial and temporal interpolations of Global Data Assimilation System (GDAS) model runs between $0700 \mathrm{Z}$ and $1200 \mathrm{Z}$ (the approximate time the aircraft was collecting data). The models provide adequate estimates for the storm center but are not used as wind direction ground truth for either sensor, as we describe below. The track of the aircraft was offset in the direction of storm motion before the overpass and in the opposite direction after the overpass, by the distance difference between the estimated storm centers at aircraft-measurement time and satellite-measurement time. The nearest Sentinel-1B measurement to the resulting location is selected for collocation. Figure 2 shows a schematic diagram of this location translation procedure.

Since the IWRAP C-band radar has a range of incidence angles to choose from, along each point in the collocated flight track the IWRAP measurements with an EIA within $\pm 1^{\circ}$ of that of the selected Sentinel-1B observation was selected. The NRCS measurements were then averaged into the same alongtrack cells that the aircraft positions are. In the following analysis, we assume both the magnitude and direction of the winds do not change significantly. Though there were most certainly some small-scale changes between the times the aircraft and satellite instruments sampled the storm, they do not affect the conclusions drawn from the comparison. While this is not possible to prove unequivocally, the auxiliary data shown below support this assumption. The dropsonde wind speeds and directions match the corresponding GDAS-interpolated $10 \mathrm{~m}$ winds relatively well. The GDAS values are interpolated from the model runs at $0600 \mathrm{Z}$ and $1200 \mathrm{Z}$, which is a greater time span than the aircraft flight. This suggests stability in the surface wind field, the primary driver of the NRCS shown in the rest of the paper. Finally, the National Weather Service (NWS)/National Centers for Environmental Prediction (NCEP) Ocean Prediction Center
(OPC) Atlantic surface analyses valid at $0600 \mathrm{Z}$ and $1200 \mathrm{Z}^{1}$ only show a difference in central pressure of the storm of 1 mbar, which is a negligible change.

\section{Calibration}

For most of the aircraft flight time, Sentinel-1B and IWRAP were not observing the ocean at the same azimuth in the same direction, which results in differences in the measured NRCS that are not due to instrument or processing differences. We compensated for this by using a geophysical model function (GMF) to predict differences between the two wind-relative observation angles, and have applied the difference to the Sentinel-1B NRCS. Note that this does not remove the wind direction effect on the observed NRCS. When showing the Sentinel-1B NRCS after compensating for azimuth look angle in the following results, they are labeled "ALAC." A more detailed description of this processing step follows.

After averaging IWRAP NRCS in the alongtrack dimension, wind directions retrieved from the Sentinel-1B images [1] were also collocated with the alongtrack cells. Both the IWRAP Cband azimuth and the Sentinel-1B azimuth were subtracted from these wind directions to obtain a wind-relative azimuth. Using these relative wind directions, the mean incidence angles of each sensor in the alongtrack bin, and SFMR wind speeds as the input to the CMOD5.h GMF [10], modeled NRCS ratios of the two sensor geometries were computed and applied to the Sentinel-1B VV NRCS. In other words, for each alongtrack bin the quantity

$$
\Delta \sigma_{\mathrm{ALAC}}^{0}=\frac{\sigma_{\mathrm{CMOD} . \mathrm{h}}^{0}\left(\mathrm{U}_{10 \mathrm{~N}}, \phi_{\text {IWRAP }}, \theta\right)}{\sigma_{\mathrm{CMOD} 5 . \mathrm{h}}^{0}\left(\mathrm{U}_{10 \mathrm{~N}}, \phi_{\mathrm{S} 1 \mathrm{~B}}, \theta\right)}
$$

was computed for NRCS in linear units and multiplied with the Sentinel-1B NRCS, where $U_{10 N}$ is the $10 \mathrm{~m}$ equivalent-neutral wind speed reported by SFMR, $\phi$ is the wind-relative azimuth angle of each sensor, and $\theta$ is the incidence angle observed by both sensors. This has the effect of rotating the Sentinel1B observation angle to match IWRAP and leaves any nongeophysical effects intact. Since there is not much wind direction dependence at cross-polarization [5], this step was not performed for the VH NRCS.

Ground calibration of IWRAP was performed before and after the flight experiment campaign in order to measure the receiver gain and transmit power levels. Because this does not include the antenna, a final, constant calibration offset is determined from the sea-surface NRCS. The calibration offsets change between installations due to radar reconfiguration, maintenance, and the simple fact that disconnecting cables for platform deintegration or ground calibration creates a slightly different measurement path.

Wind speed retrievals from SFMR were performed using coldweather corrections to the tropical-weather GMF [11] to obtain reference wind speeds collocated with IWRAP. SFMR wind speeds from 22 to $24 \mathrm{~m} \mathrm{~s}^{-1}$ were selected along with IWRAP NRCS measurements at incidence angles of $29^{\circ}$ to $31^{\circ}$ from this flight. The CMOD5.h GMF was used with wind directions as above to compute the true NRCS. The incidence angle range is

\footnotetext{
${ }^{1}$ See https://www.ncei.noaa.gov/thredds/catalog/ncep_charts_new_charts_ ocean_anl/201702/20170206/catalog.html
} 
within the valid range of CMOD5.h (which inherits its limits from CMOD5.n [12]) and the C-band fan-beam of IWRAP during level flight. The wind speeds were chosen such that they would target a large area at the beginning of the collocation region and be in a valid range for both SFMR and CMOD5.h. The median of the differences between the GMF and measured NRCS at VV-polarization was added to the IWRAP VV NRCS. The HH calibration adjustment is estimated by computing the VV-only CMOD5.h and applying the wind-speed- and EIAdependent polarization ratio from [13].

Assuming these small, constant calibration offsets are from the antenna connections, each adjustment is a result of the electromagnetic waves propagating both into and out of each polarization port. Therefore, the cross-polarization calibration adjustment is the sum (in decibels) of half of the co-polarized offsets (the geometric mean in linear units). We do not rely on a VH GMF for calibration because none are mature enough in our opinion, and it is more appropriate to correct for the source of the error (in this case, the V- and H-polarized antenna connections) than the resulting behavior (VH NRCS).

All of these calibration adjustments are on the same order of magnitude as in previous years (e.g., [5], [14]). HH-polarization results are not shown here since Sentinel-1B was not measuring $\mathrm{HH}$ NRCS at the time.

This procedure neither causes the IWRAP NRCS to track SFMR wind speeds nor does it affect the incidence angle dependence; it only ties the means of the IWRAP measurements in the small wind speed range to a fixed value. Thus the independence of the two systems are retained. However, there still may remain a constant offset to the true NRCS in both VV and $\mathrm{VH}$.

Polarization mixing is the effect that occurs when rotating the antenna's electric field away from perpendicular or parallel to the plane of the ocean. Some amount of HH-polarized NRCS is "mixed" with the VV measurements, and co-polarized NRCS likewise contaminate cross-polarized measurements. The correction for this phenomena is most noticeable in the $\mathrm{VH}$ measurements. In the following results, we have calculated and removed the contribution to measured VH NRCS due to polarization mixing using the aircraft's pitch, the antenna mount angle, and (3) in [5]:

$$
\sigma_{V H}^{0}{ }^{\prime}=\sigma_{V H}^{0}+\left(\sigma_{V V}^{0}+\sigma_{H H}^{0}\right) \sin ^{2} \gamma \cos ^{2} \gamma,
$$

where the prime symbol indicates the measured quantity and $\gamma$ is the negative value of the aircraft pitch angle. The pure NRCS is simulated using the same co-polarized geophysical model functions used for calibration. This adjustment is less than $0.1 \mathrm{~dB}$ for more than $95 \%$ of the $\mathrm{VH}$ data.

\section{Results And Discussion}

Figure 3 shows the IWRAP and Sentinel-1B NRCS as a function of time for VV-polarization (top panel) and VH-polarization (bottom). In the bottom part of each panel, the SFMR $10 \mathrm{~m}$ equivalent-neutral wind speed is shown along with the same wind speed estimates from GPS dropwindsondes [15] and GDAS. As the dropsondes measure a profile, an averaging and scaling method (WL150) has been applied to estimate the equivalentneutral wind speed at $10 \mathrm{~m}$. This algorithm is described in [5].
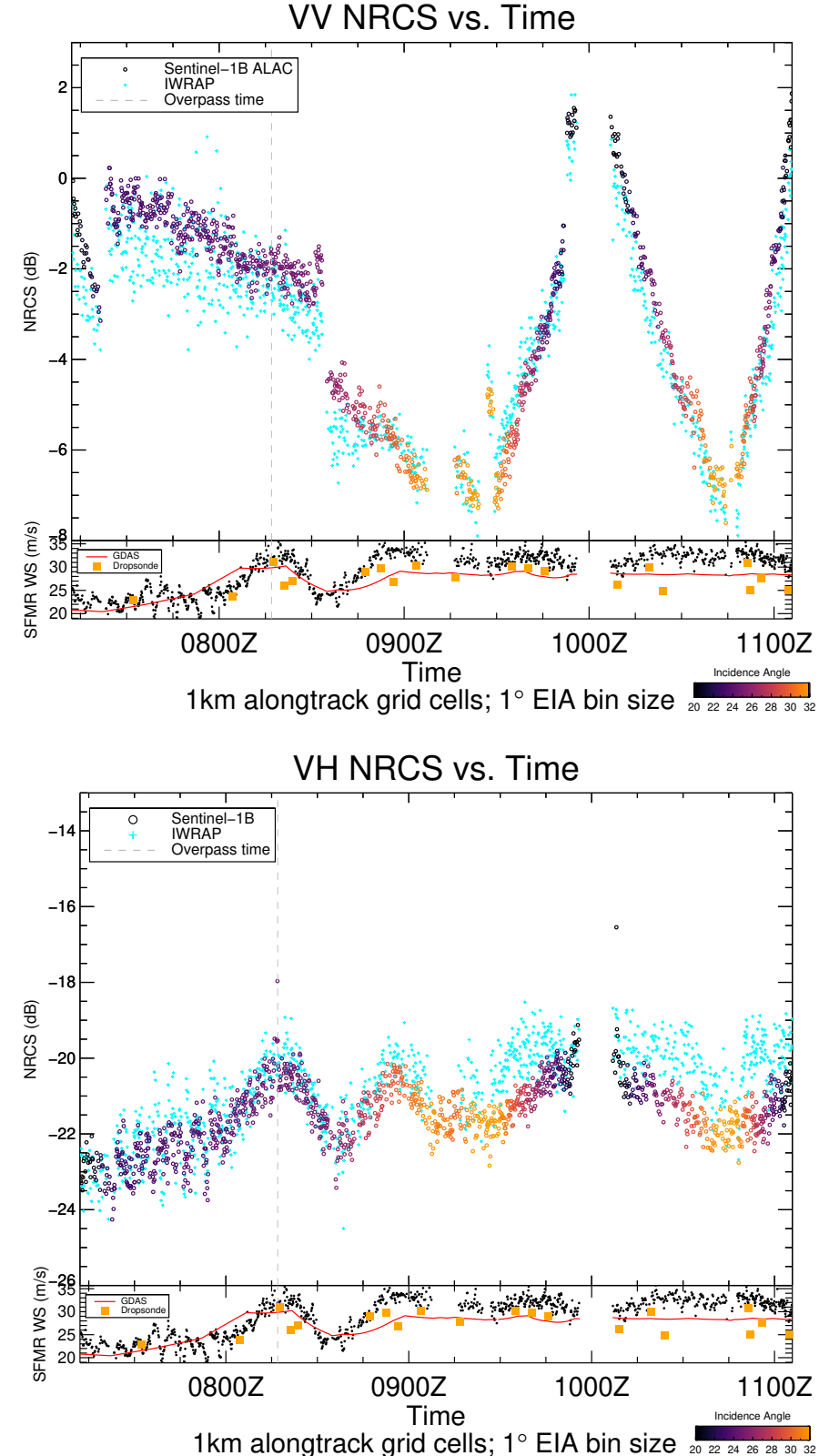

Figure 3. NRCS (in decibels) as a function of time during the flight on 6 Feb 2017. The upper panel shows VV-polarization and the lower panel shows VHpolarization. The main part of each panel shows the IWRAP NRCS as crosses and the Sentinel-1B NRCS as empty circles colored by the incidence angle. Within each panel, the lower part shows the wind speed retrieved from SFMR as well as the surface wind speed estimation from dropsondes.

Some data appear missing due to being outside the swath (at approximately 1000Z; see also Figure 1) or during large-angle turns or $360^{\circ}$ orbits (at approximately 0905-0910Z and 1045Z), where the SFMR retrievals are not fully validated.

The dropsonde surface wind speed estimates are slightly lower than SFMR wind speeds in general. A few report much lower speeds than SFMR, but this has been observed in other comparisons using a relatively small number of dropsondes [5] and do not necessarily indicate errors in SFMR retrievals. It is possible that SFMR wind speeds are too high due to imperfect cold-weather corrections. However, the conservative approach for the present analysis is to use the SFMR wind speeds as 
they are. Reducing the wind speed used in (1) increases the adjustment due to ALAC. This increases the slope of the VV sensor ratios (top panels of Figures 4 and 5) and results in a greater difference between subswaths.

The dropsondes appear to align with the interpolated GDAS wind speeds relatively well, but GDAS does not adequately represent the higher gradients. This is not only apparent compared to SFMR wind speeds, but also with the two VH NRCS measurements.

As can be seen in Figure 1 and the coloring of Figure 3, the track of the aircraft proceeded generally towards an increasing incidence angle until approximately 0930Z. At this time, the aircraft turned East, exits the swath near 1000Z, and turned back West again. Outside of the swath is below the lowest EIA observed by Sentinel-1B, so during this Eastbound leg the VV NRCS can be seen to have increased with time as EIA decreases. After having reached the Westernmost point at approximately 1045Z, the aircraft turned East again. From 0900 to $1100 Z$ the wind speed remained relatively constant, so changes in the $\mathrm{VH}$ NRCS measured by both sensors during this time span are due to the weak EIA sensitivity.

The VV-polarized NRCS are not obviously correlated with wind speed, shown in the lower part of each panel of Figure 3, but are strongly influenced by EIA and wind-relative look angle. For example, from approximately $0715 \mathrm{Z}$ to $0830 \mathrm{Z}$ the EIA changed from $23^{\circ}$ to $25^{\circ}$ and the wind-relative azimuth changed from $180^{\circ}$ to $140^{\circ}$. There is sensitivity of VV NRCS to wind speed at these speeds, but it is masked by the stronger sensitivity to EIA.

Though CMOD5.h may not be absolutely precise at these low incidence angles (due to limitations in the development of CMOD5.n; see below for further discussion), its behavior is useful for understanding the $\mathrm{VV}$ response to changes in EIA and azimuth in general. For the values of wind speed, EIA, and wind-relative azimuth of the sensors during this time, CMOD5.h predicts 1.5 to $2 \mathrm{~dB}$ of NRCS decrease. This is nearly the same amount of change observed by both IWRAP and Sentinel-1B. Since differences with IWRAP due to azimuth angle have been removed from the Sentinel-1B co-polarized data, any remaining difference between IWRAP and Sentinel-1B may be primarily attributed to calibration errors in IWRAP or Sentinel-1B.

In contrast to $\mathrm{VV}$, the $\mathrm{VH}$ NRCS measurements from both sensors follow the same trend as the SFMR wind speed and are weakly influenced by EIA. The VH NRCS tracks the changes in wind speed despite the changes in EIA and wind-relative azimuth.

Note that two elevated Sentinel-1B VH samples at the collocation time and near 1010Z, visible near the $-18 \mathrm{~dB}$ level in the bottom panel of Figure 3, do not seem to be from the ocean surface backscatter but instead from the aircraft. Since these don't appear in the co-polarized channel, it isn't obvious whether they are from interaction with the body of the airplane or some other active transmitter on the airplane. The aircraft interference with the Sentinel-1B measurement exists across the satellite track of subswath 1 , so it is the closest collocated measurement in two different locations.

Figure 4 shows the ratio of IWRAP to Sentinel-1B NRCS in linear units as a function of Sentinel-1B antenna elevation
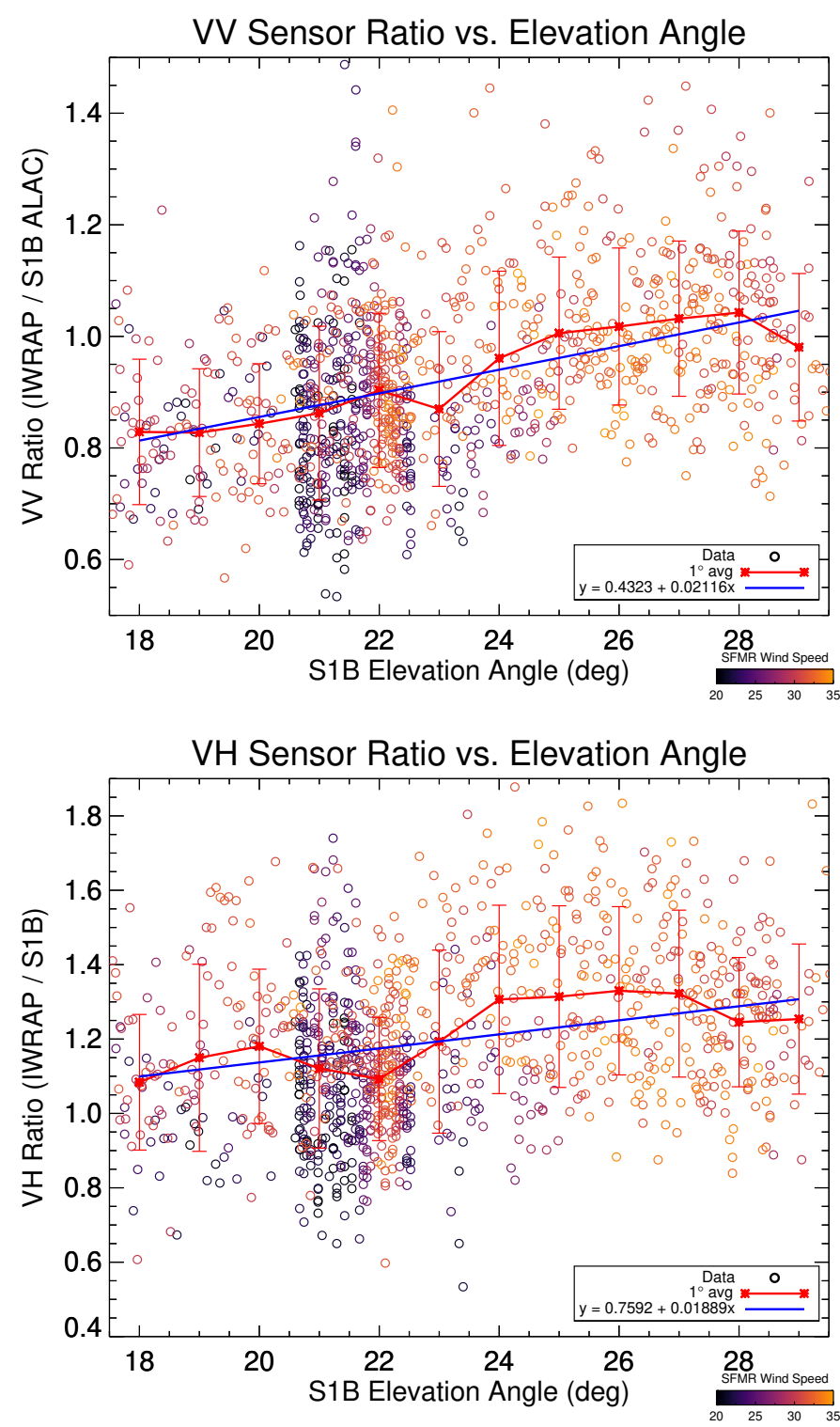

Figure 4. NRCS ratio (in linear units) between IWRAP and Sentinel-1B as a function of the Sentinel-1B elevation angle during the flight on 6 Feb 2017. The upper panel shows VV-polarization and the lower panel shows VH-polarization. The empty circles show the raw data colored by SFMR wind speeds and the asterisks connected by a solid line are means and sample standard deviations within $1^{\circ}$ elevation angle bins. The solid line shows a linear fit to the means, with the equation given in the legend.

angle. The individual comparison points are colored by SFMR wind speed to show that any trend is not due to wind speed contributions. Figure 5 is the same but for the data prior to ESA-reprocessing made available only to the Expert Support Laboratory working on Sentinel-1 in Spring 2018-in other words, the data available to the public. A linear fit was performed to the means.

Vertical offsets from a ratio of 1 indicate that IWRAP NRCS is larger (positive offset) or smaller (negative offset) than Sentinel1B NRCS. There is a slight trend in both VV and VH ratio, and the two slopes are nearly the same. This was not just an effect of time difference; as can be seen in Figure 3, the elevation angle being used varied throughout the flight independent of time. There is not an obvious surface wind speed dependence, 

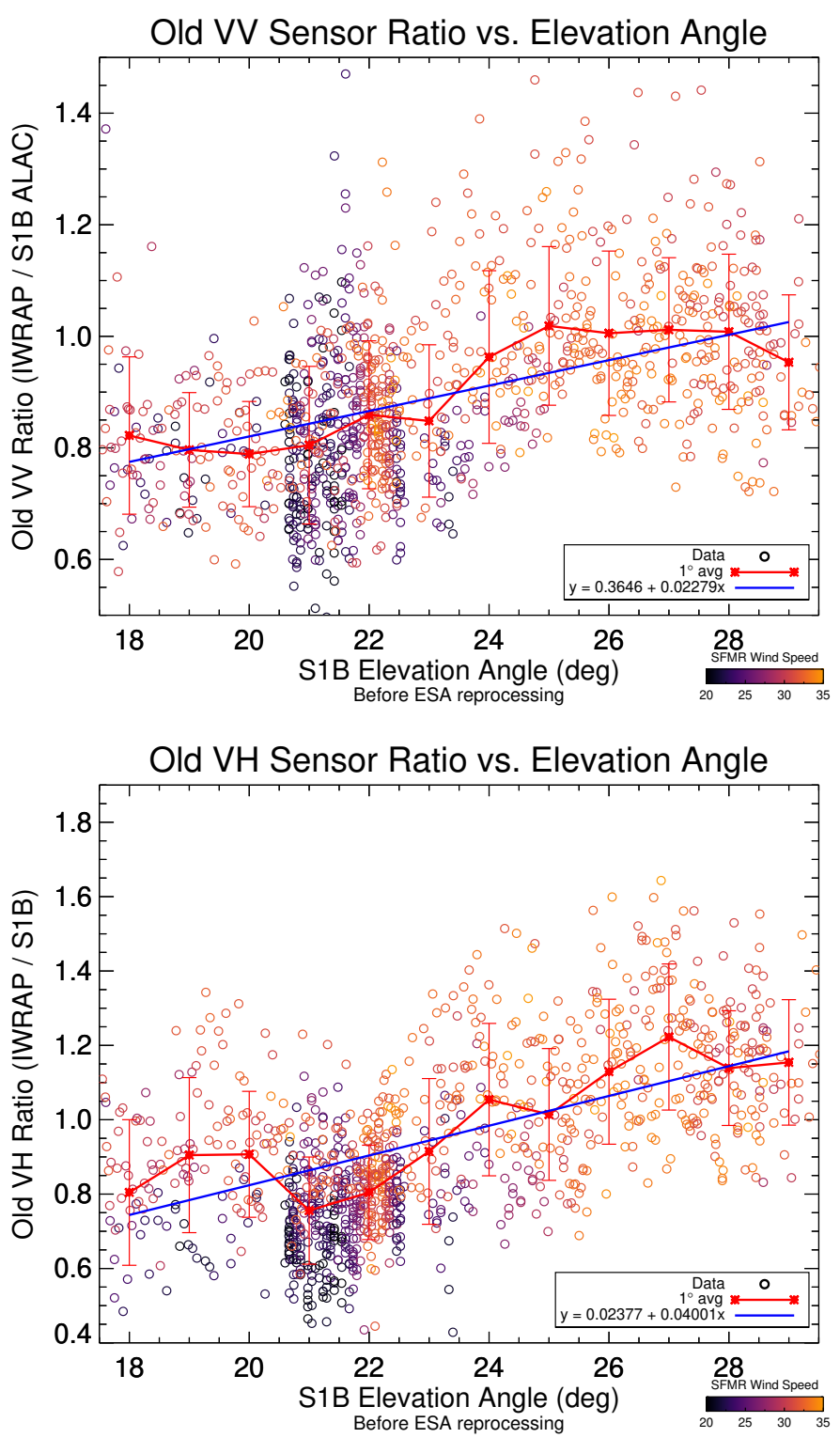

Figure 5. NRCS ratio (in linear units) between IWRAP and Sentinel-1B as a function of the Sentinel-1B elevation angle during the flight on 6 Feb 2017 prior to the ESA reprocessing in Spring 2018. The upper panel shows VV-polarization and the lower panel shows VH-polarization. The empty circles show the raw data colored by SFMR wind speeds and the asterisks connected by a solid line are means and sample standard deviations within $1^{\circ}$ elevation angle bins. The solid line shows a linear fit to the means, with the equation given in the legend.

as retrieved from SFMR.

To rule out a bias in the IWRAP NRCS with incidence angle (due to knowledge of the gain pattern, surface area calculation, or errors resulting from incorrect antenna attitude), we show the ratio of modeled NRCS to both IWRAP and S1B NRCS as a function of Sentinel-1B elevation angle in Figure 6. The NRCS GMF chosen was CMOD5.h; we used SFMR retrievals as the wind speed input and the same wind direction described in Section III. Comparisons with IWRAP alongtrack-averaged data and $1^{\circ}$ means are shown as empty triangles and asterisks, respectively. The same is shown for Sentinel-1B as empty circles and filled circles. A value below 1 indicates the sensor is reporting higher NRCS than modeled.

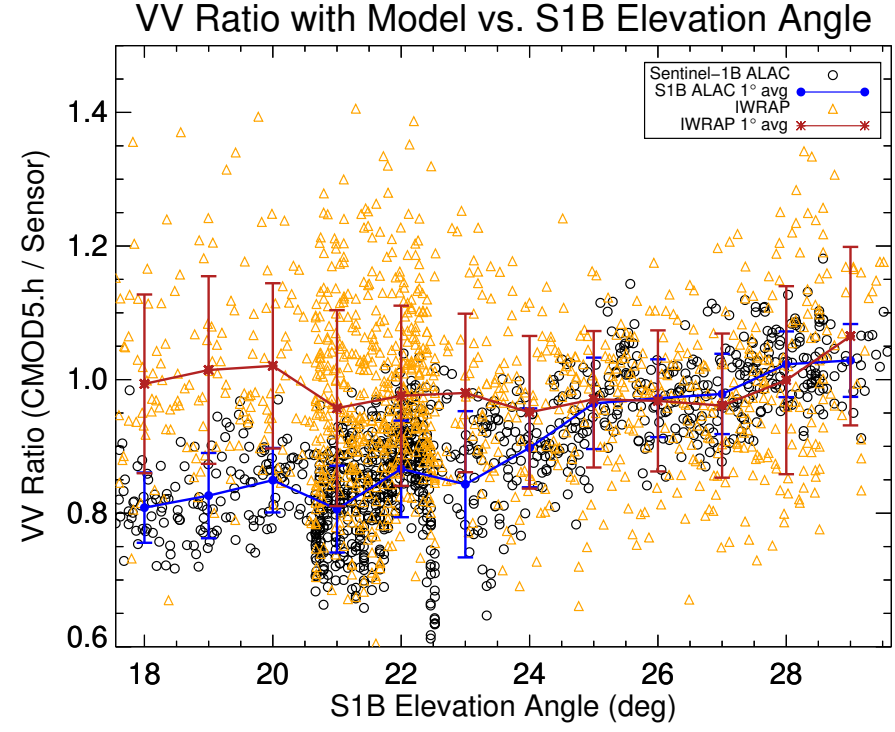

Figure 6. VV-polarized NRCS ratio (in linear units) between the CMOD5.h GMF and each sensor as a function of the Sentinel-1B elevation angle during the flight on 6 Feb 2017. Empty circles show the Sentinel-1B data and empty triangles show the IWRAP data. Filled circles show the $1^{\circ}$ average of the Sentinel-1B data and asterisks show the same for IWRAP. Surface wind speeds were from SFMR and wind directions were retrieved from the Sentinel-1 image.

One may expect that IWRAP NRCS be nearly 1 in the mean in Figure 6 due to the calibration procedure. While it is close (within 1 sample standard deviation), it appears to be a little low. Recall that IWRAP was calibrated at $30^{\circ}$ incidence $\left( \pm 1^{\circ}\right)$, which corresponds approximately to the 25.5 to $27.5^{\circ}$ elevation angle range; the CMOD5.n (and, by extension, CMOD5.h) GMF at incidence angles below $27^{\circ}$ was developed using a different sensor than for the higher incidence angles. Recent GMF work [16] have observed and compensated for uncertainty in CMOD5.n at low incidence angles (for example, see Fig. 3 of [16]). Considering this, the NRCS measured by IWRAP is within an expected margin of error of CMOD5.h. The IWRAP data at low incidence angles (18 to $20^{\circ}$ ) suggest that the lowincidence angle component of CMOD5.n should be revisited, as has been done with CMOD7 [16].

We do not use CMOD7 here because it was not developed specifically for high winds as CMOD5.h was and it uses stressequivalent wind speeds as the surface wind speed. This is not the same wind speed as the SFMR or dropsonde reports. Since it only causes a small vertical shift in the NRCS of Figure 6 (not shown), we use CMOD5.h for this figure and the ALAC.

There is definitely a strong trend in the Sentinel-1B VV NRCS ratio, while the IWRAP VV measurements show little difference compared to the model. Figure 6 shows that the VV observations in Figures 4 and 5 are not due to a bias in IWRAP. A larger scatter in the IWRAP data is noticeable despite the alongtrack averaging on the same order as the Sentinel-1B averaging. This is due to the smaller cross-track size.

Figure 7 shows the VH NRCS as a function of incidence angle for IWRAP and Sentinel-1B. To make a comparison of the incidence angle dependence of the VH NRCS between the two sensors, the NRCS data where SFMR wind speeds are higher than $30 \mathrm{~m} \mathrm{~s}^{-1}$ have been averaged in $1^{\circ}$ EIA bins and plotted as 

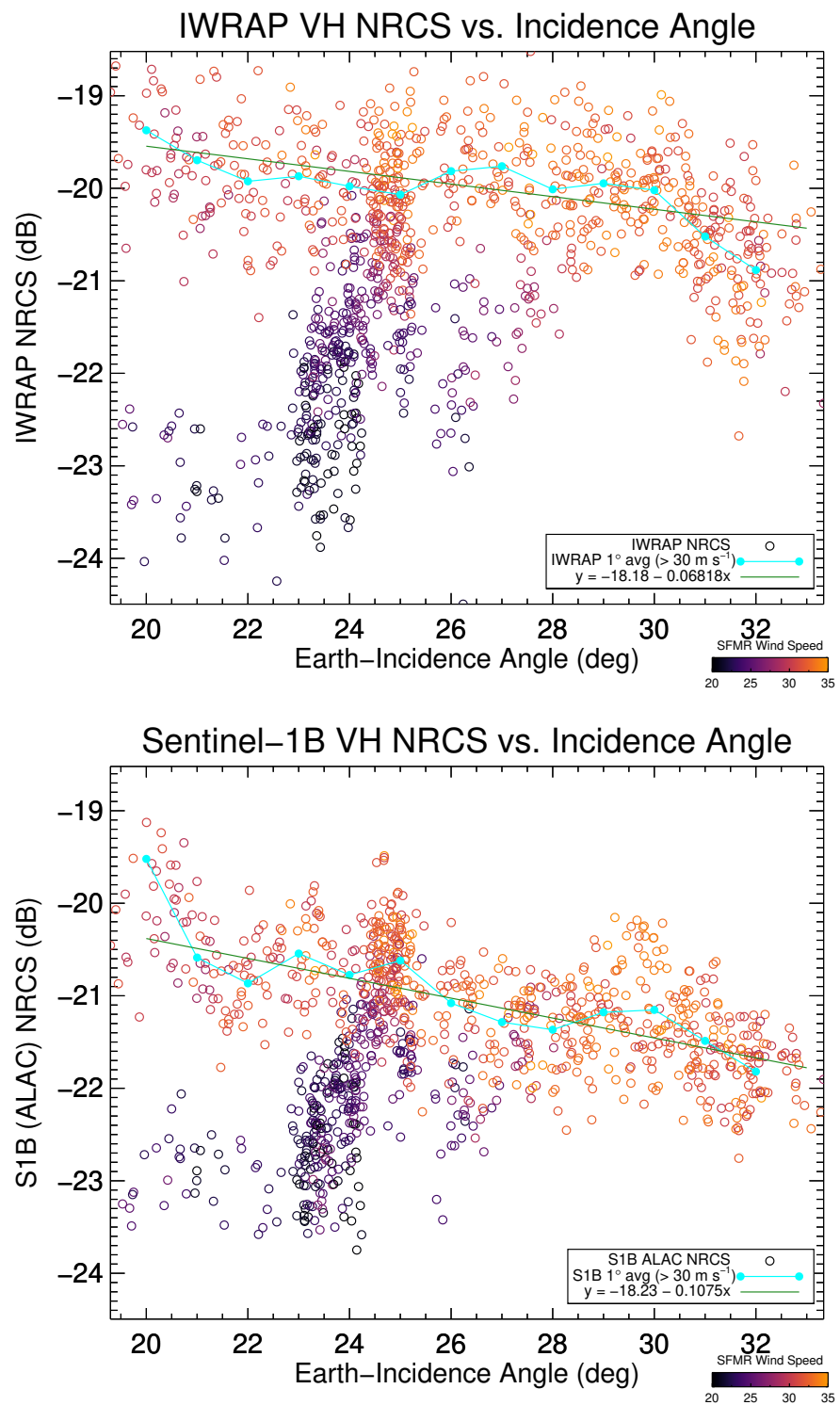

Figure 7. VH NRCS vs. Earth-incidence angle for the two sensors from the flight on 6 Feb 2017. The upper panel shows IWRAP and the lower panel shows Sentinel-1B. The points are colored according to SFMR wind speed. Filled circles connected by a solid line are the means of the data points where the SFMR wind speed is higher than $30 \mathrm{~m} \mathrm{~s}^{-1}$ in $1^{\circ}$ elevation angle bins. The solid line shows a linear fit to the means, with the equation (fit in decibels) given in the legend.

filled circles. The darker colored points were measured at lower surface wind speeds. The strong wind speed sensitivity can be observed in the difference between these and the values above $30 \mathrm{~m} \mathrm{~s}^{-1}$

Though the connected points of Figures 6 and 7 suggest a smooth transition between Sentinel-1B subswaths, this may not be the case. The subswath transition occurs at approximately $24^{\circ}$ elevation. A closer look at Figure 6 reveals a good match (ratio of 0.9 to 1 ) between modeled and measured NRCS above $24^{\circ}$ and a clear lower ratio (below 0.8 ) below $23^{\circ}$. It is not clear whether or not the NRCS in subswath 2 are mis-aligned, but it is apparent that the measurements in subswath 1 (low elevation angles) are biased high.

As noted in Section III, the absolute calibration level of the
IWRAP VH channel may not be correct. Based on Figure 6, we expect the higher incidence angles to match better in power. Comparing with Figure 4, the IWRAP VH channel may be $1.3 \mathrm{~dB}$ too high. However, the relative calibration is reliable and the trends shown in Figure 7 can be analyzed in light of these facts. Despite the same geophysical scene and the same decreasing trend of VH NRCS with increasing EIA, Sentinel-1B clearly shows a stronger dependence of VH NRCS on EIA. This is consistent with our conclusions about VV NRCS drawn from Figure 6: that the Sentinel-1B subswath 1 measurements are too high compared to IWRAP measurements.

Some papers using RADARSAT-2 SAR data have concluded that no incidence angle sensitivity exists for VH NRCS [13], [17]. However, both sensors show a weak VH NRCS dependence on EIA, which is consistent with other studies (e.g., [1], [18]-[20] and theory (e.g., [21], [22]). It is also consistent with prior IWRAP-based findings [23], though to a lesser degree than [5]. The IWRAP VH data in Figure 7 show a more subtle slope than those from Sentinel-1B.

\section{Summary}

The data collected from the NOAA WP-3D flight experiment on 6 Feb 2017 contributes to at least two of the objectives of the NOAA Ocean Winds flight experiment program: development and improvement of space-based ocean surface vector wind (OSVW) products, and testing of new remote sensing technologies for future satellite missions.

The data presented here further demonstrate the utility of having both VV- and VH-polarized channels on a scatterometer or SAR. With sensitivities to different ocean parameters, both polarizations further constrain wind vector retrievals. The second-generation MetOp scatterometer (MetOp-SG SCA) will have both VV and VH capabilities, and this collocated dataset clearly demonstrates the strong sensitivity of the VH NRCS signal to wind speed from both aircraft and satellite sensors. The data also show the relative insensitivity of $\mathrm{VH}$ to wind direction, a feature that should prove useful for wind speed retrievals from the scatterometer on MetOp-SG. Since only the antenna perpendicular to the flight path on each side of the spacecraft is planned to have the cross-polarized channel, this insensitivity may reduce the need for multiple looks at a wind vector cell from different directions. This analysis also confirms that there is no or very little sensitivity to the incidence angle for VH NRCS.

In the course of writing this manuscript ESA reprocessed the Sentinel-1B data presented here for use by the Expert Support Laboratory working on Sentinel-1. A significant trend of S1B VH NRCS with elevation angle, compared to IWRAP NRCS, was improved and a step at subswath edges was reduced. However, VV NRCS largely remained the same. The VH NRCS still show a small bias in one of the subswaths. The choice of ocean calibration model functions impact the absolute level of the IWRAP VH NRCS. There may be a trend in the whole range of antenna elevation angles or it may be limited to a discontinuity at the subswath boundary.

The VV NRCS show a similar bias or trend as the VH do, but inaccuracies in surface wind speed or direction would lead to a different ratio between Sentinel-1B and IWRAP. There is 
better agreement with IWRAP and modeled VV NRCS generated from SFMR wind speeds and Sentinel-1B wind directions in subswath 2, which suggests that there are processing-related problems in subswath 1 .

Overall, this paper confirms that getting both calibrated $\mathrm{VH}$ NRCS from satellites and intercalibrated measurements from different remote sensing systems remains a challenge. A careful analysis and monitoring of the Sentinel-1 SAR across the swath over permanent scatterers, such as a rain forest (similar to [24]) is certainly needed to fully characterize the antenna gain pattern. The same is true for the noise annotated in the Level-1 products and are confirmed by the recent updates in the ESA Sentinel-1 Level-1 processor.

Interestingly, this study reveals that the inconsistency between IWRAP and Sentinel-1 or between CMOD5.n (VV GMF) and Sentinel-1 are more pronounced at low incidence angles where no ASCAT data exist to define the VV GMF. This inconsistency will impact the wind speed retrieval derived from VV NRCS at low incidence angles from Sentinel-1. This point also suggests a closer look be taken at the VV GMF that is to be used for the next-generation MetOp-SG SCA instrument, which will have lower incidence angles than the existing ASCATs.

Finally, regarding VH NRCS: if used for wind speed retrieval, the choice of the VH GMF may depend on the sensor. In particular, the VH GMF proposed from RadarSat-2 or Sentinel-1 data may not be appropriate for MetOp-SG SCA.

\section{ACKNOWLEDGMENT}

The authors gratefully acknowledge the crew of N42RF and the generous logistical and technical support of the NOAA NOAA Aircraft Operations Center (AOC) in Lakeland, FL. F. Said (GST) made observations that were useful in directing some of the analysis. This manuscript contains modified Copernicus Sentinel data [2017].

\section{REFERENCES}

[1] A. A. Mouche, B. Chapron, B. Zhang, and R. Husson, "Combined Co- and Cross-Polarized SAR Measurements Under Extreme Wind Conditions," IEEE Trans. Geosci. Remote Sens., pp. 6746-6755, 2017. DOI: 10.1109/TGRS.2017.2732508.

[2] Y. Zhao, A. A. Mouche, B. Chapron, and N. Reul, "Direct Comparison Between Active C-Band Radar and Passive L-Band Radiometer Measurements: Extreme Event Cases," en, IEEE Geosci. Remote Sens. Lett., vol. 15, no. 6, pp. 897-901, Jun. 2018. DoI: 10.1109/LGRS.2018.2811712.

[3] F. D. Zan and A. M. Guarnieri, "TOPSAR: Terrain Observation by Progressive Scans," IEEE Trans. Geosci. Remote Sens., vol. 44, no. 9, pp. 2352-2360, Sep. 2006. DoI: 10.1109/TGRS. 2006.873853

[4] D. E. Fernandez, E. M. Kerr, A. Castells, J. R. Carswell, S. J. Shaffer, P. S. Chang, P. G. Black, and F. D. Marks, "IWRAP: The Imaging Wind and Rain Airborne Profiler for remote sensing of the ocean and the atmospheric boundary layer within tropical cyclones," IEEE Trans. Geosci. Remote Sens., vol. 43, no. 8, pp. 1775-1787, Aug. 2005. Dor: 10.1109/TGRS.2005.851640.

[5] J. W. Sapp, S. O. Alsweiss, Z. Jelenak, P. S. Chang, S. J. Frasier, and J. Carswell, "Airborne Co-polarization and CrossPolarization Observations of the Ocean-Surface NRCS at CBand," IEEE Trans. Geosci. Remote Sens., vol. 54, no. 10, pp. 5975-5992, Jul. 2016. DoI: 10.1109/TGRS.2016.2578048.
[6] J. Sapp, S. Alsweiss, Z. Jelenak, and P. Chang, "Stepped Frequency Microwave Radiometer Retrieval Error Characterization," in Geoscience and Remote Sensing Symposium (IGARSS), 2017 IEEE International, Fort Worth, TX, 2017, pp. 271-274. DoI: 10.1109/IGARSS.2017.8126947.

[7] C. Lin, F. Fois, A. Østergaard, M. Loiselet, Q. Garcia, A. Trastoy, P. Magnusson, P. Dimming, and M. Petersson, "Antenna Development for MetOp Second Generation Wind Scatterometer," in Proc. 9th EuCAP, Lisbon, Portugal, Apr. 2015.

[8] P. Magnusson, P. Dimming, C. Lin, and A. Østergaard, "A thermally stable dual-polarized waveguide array," in Proc. 9th EuCAP, Lisbon, Portugal, Apr. 2015.

[9] ProSensing, Stepped Frequency Microwave Radiometer (SFMR) - ProSensing, http://www.webcitation.org/query?url=http $\% 3 \mathrm{~A} \% 2 \mathrm{~F} \% 2 \mathrm{Fwww}$.prosensing.com $\% 2$ Fcrb-product $\% 2 \mathrm{Fsfmr} \% 2 \mathrm{~F} \&$ date $=2018-11-28$, 2018.

[10] S. Soisuvarn, Z. Jelenak, P. S. Chang, S. O. Alsweiss, and Q. Zhu, "CMOD5.H-A High Wind Geophysical Model Function for C-Band Vertically Polarized Satellite Scatterometer Measurements," IEEE Trans. Geosci. Remote Sens., vol. 51, no. 6, pp. 3744-3760, Jun. 2013. DOI: 10.1109/TGRS.2012.2219871.

[11] J. W. Sapp, S. O. Alsweiss, Z. Jelenak, P. S. Chang, and J. Carswell, "Stepped Frequency Microwave Radiometer WindSpeed Retrieval Improvements," Remote Sens., vol. 11, no. 3, p. 214, Jan. 2019. DOI: 10.3390/rs11030214.

[12] A. Verhoef, M. Portabella, A. Stoffelen, and H. Hersbach, "CMOD5.n - the CMOD5 GMF for neutral winds," EUMETSAT, Tech. Rep. SAF/OSI/CDOP/KNMI/TEC/TN/165, May 2008.

[13] B. Zhang, W. Perrie, and Y. He, "Wind speed retrieval from RADARSAT-2 quad-polarization images using a new polarization ratio model," J. of Geophys. Res.: Oceans, vol. 116, no. C8, 2011. DOI: 10.1029/2010JC006522.

[14] J. W. Sapp, S. J. Frasier, J. Dvorsky, P. S. Chang, and Z. Jelenak, "Airborne Dual-Polarization Observations of the Sea Surface NRCS at C-Band in High Winds," IEEE Geosci. Remote Sens. Lett., vol. 10, no. 4, pp. 726-730, Jul. 2013. DoI: 10.1109/LGRS. 2012.2220118.

[15] T. F. Hock and J. L. Franklin, "The NCAR GPS Dropwindsonde," en, Bull. Am. Meteorol. Soc., vol. 80, no. 3, pp. 407-420, Mar. 1999. DOI: 10.1175/1520-0477(1999)080<0407:TNGD>2.0.CO; 2.

[16] A. Stoffelen, J. A. Verspeek, J. Vogelzang, and A. Verhoef, "The CMOD7 Geophysical Model Function for ASCAT and ERS Wind Retrievals," IEEE J. Sel. Top. Appl. Earth Obs. Remote Sens., vol. 10, no. 5, pp. 2123-2134, May 2017. DoI: 10.1109/JSTARS. 2017.2681806.

[17] P. W. Vachon and J. Wolfe, "C-Band Cross-Polarization Wind Speed Retrieval," IEEE Geosci. Remote Sens. Lett., vol. 8, no. 3, pp. 456-459, 2011. DoI: 10.1109/LGRS.2010.2085417.

[18] G.-J. van Zadelhoff, A. Stoffelen, P. W. Vachon, J. Wolfe, J. Horstmann, and M. Belmonte Rivas, "Retrieving hurricane wind speeds using cross-polarization C-band measurements," Atmos. Meas. Tech., vol. 7, no. 2, pp. 437-449, Feb. 2014. Dor: 10. 5194/amt-7-437-2014.

[19] F. Fois, P. Hoogeboom, F. Le Chevalier, and A. Stoffelen, "Future Ocean Scatterometry: On the Use of Cross-Polar Scattering to Observe Very High Winds," IEEE Trans. Geosci. Remote Sens., vol. 53, no. 9, pp. 5009-5020, Sep. 2015. DoI: 10.1109/TGRS. 2015.2416203.

[20] J. Horstmann, S. Falchetti, C. Wackerman, S. Maresca, M. J. Caruso, and H. C. Graber, "Tropical Cyclone Winds Retrieved From C-Band Cross-Polarized Synthetic Aperture Radar," IEEE Trans. Geosci. Remote Sens., vol. 53, no. 5, pp. 2887-2898, Apr. 2015. DOI: 10.1109/TGRS.2014.2366433.

[21] A. Voronovich and V. Zavorotny, "Depolarization of microwave backscattering from a rough sea surface: Modeling with smallslope approximation," IEEE, Jul. 2011, pp. 2033-2036. Dor: 10. 1109/IGARSS.2011.6049530. 
[22] P. A. Hwang, B. Zhang, J. V. Toporkov, and W. Perrie, "Comparison of composite Bragg theory and quad-polarization radar backscatter from RADARSAT-2: With applications to wave breaking and high wind retrieval," J. Geophys. Res., vol. 115, no. C8, Aug. 2010. Dor: 10.1029/2009JC005995.

[23] J. Sapp, Z. Jelenak, P. Chang, and S. Frasier, "C-Band CrossPolarization Ocean Surface Observations in Hurricane Matthew," in 2018 IEEE International Geoscience and Remote Sensing Symposium (IGARSS), Jul. 2018, pp. 5595-5598. DoI: 10.1109/ IGARSS.2018.8519433.

[24] J. Figa-Saldaña, J. J. Wilson, E. Attema, R. Gelsthorpe, M. R. Drinkwater, and A. Stoffelen, "The advanced scatterometer (ASCAT) on the meteorological operational (MetOp) platform: A follow on for European wind scatterometers," Can. J. Remote Sens., vol. 28, no. 3, pp. 404-412, 2002. DoI: 10.5589/m02-035.

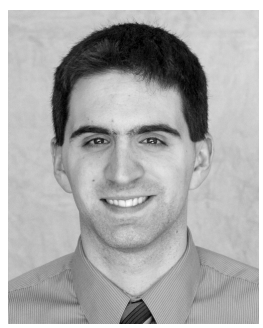

Joseph W. Sapp (Member, IEEE) received the B.S degree in electrical engineering from The Pennsylvania State University, University Park, PA, USA in 2006 and the Ph.D. degree in electrical and computer engineering from the University of Massachusetts Amherst, Amherst, MA, USA in 2015.

From 2006 to 2009 he was a Project Electrical Engineer with Lutron Electronics Co., Inc., Coopersburg, PA, USA, during which he was involved in the embedded software design of multiple commercial products. In 2009 he joined the Microwave Remote Sensing Laboratory, University of Massachusetts Amherst to complete his graduate work. During this time he had the opportunity to begin participating in flights with the National Oceanic and Atmospheric Administration (NOAA) Hurricane Hunters. Since 2015 he has been with NOAA/National Environmental Satellite, Data and Information Service (NESDIS)/Center for Satellite Applications and Research (STAR) as a Support Scientist with Global Science \& Technology, Inc. Greenbelt, MD, USA. His recent research involves using active, passive, and in situ sensors together to improve the algorithms and equipment used in remote sensing of ocean surface and atmospheric wind vectors in extreme environments.

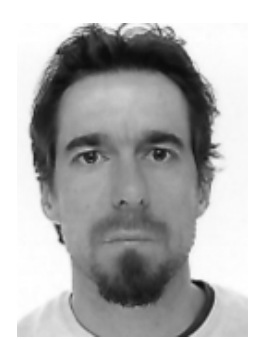

Alexis A. Mouche received the Ph.D. degree in ocean remote sensing from the Université de Versailles SaintQuentin, Versailles, France, in 2005.

He has been with the Laboratoire d'Océanographie Physique et Spatiale, Institut Francais de Recherche pour l'Exploitation de la Mer, Plouzane, France, since 2014. His research interests include the interaction of electromagnetic and oceanic waves for ocean remote sensing applications.

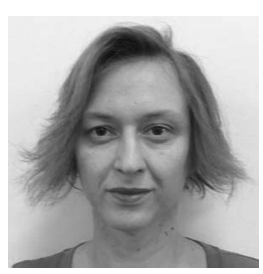

Zorana Jelenak (Member, IEEE) received the Ph.D degree in Physics from the Waikato University, Hamilton, New Zealand, in 2000.

In March 2001, she joined the Ocean Winds Team at National Oceanic and Atmospheric Administration (NOAA)/National Environmental Satellite, Data and Information Service (NESDIS) Office of Research Applications (ORA) as a University Corporation for Atmospheric Research (UCAR) visiting scientist. She is a member of the NASA Ocean Surface Winds science team, NOAA's Environmental Data Record (EDR) algorithm lead for the AMSR-2 radiometer, and a member of the NASA CYGNSS science team. Her interests are in ocean surface wind vector measurements from active and passive microwave sensors and their applicability in an operational near-real time environment, retrieval algorithm development, model function development, advanced statistical analysis, and error analysis for improved algorithm characterization.

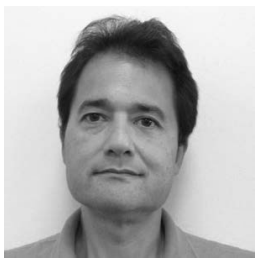

Paul S. Chang (Senior Member, IEEE) received the B.S. degree in electrical engineering from the Union College, Schenectady, NY, USA, in 1988 and the Ph.D. degree in electrical engineering in 1994 from the University of Massachusetts Amherst, Amherst, MA, USA.

Since 1994, he has been a research physical scientist with the Center for Satellite Applications and Research (STAR), National Environmental Satellite, Data and Information Service (NESDIS), National Oceanic and Atmospheric Administration (NOAA). Current activities include research and development in active and passive microwave remote sensing of the ocean surface with and emphasis on retrieval of the ocean surface wind field. Wind retrieval algorithm improvements and new product developments are pursued through the analyses of satellite and aircraft microwave remote sensing data. An emphasis is placed on transitioning research results into operational use, which involves cooperative relationships with the operational facets of NESDIS and with the National Weather Service, a primary end user of these data. Current efforts are focused on working on the METOP-ASCAT, SCATSAT, CFOSAT, CYGNSS and GCOM-W missions in addition to planning and risk reduction activities for future ocean vector winds missions.

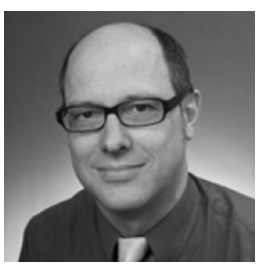

Stephen J. Frasier (Senior Member, IEEE) received the B.E.E. degree from the University of Delaware, Newark, DE, USA in 1987, and the Ph.D. degree from the University of Massachusetts Amherst, Amherst, MA, USA in 1994

From 1987 to 1990 he was with SciTec, Inc., Princeton, NJ, USA analyzing the electromagnetic and optical signatures of rocket plumes, evaluating laser detection systems, and developing data acquisition systems supporting airborne IR sensors. In August 1990 he joined the Microwave Remote Sensing Laboratory, University of Massachusetts Amherst, where his graduate work involved the development and application of digital-beamforming phased-array radar for oceanographic research applications. In 1997, he joined the faculty at the University of Massachusetts where he is currently a Professor and the Co-Director of the Microwave Remote Sensing Laboratory. From 2005 to 2006, he spent a sabbatical year with the Polytechnic University of Catalonia, Barcelona, Spain studying lidar techniques for atmospheric profiling, and in 2012, he spent a sabbatical year with Météo-France, Paris, France, evaluating performance of an X-band weather radar network being deployed in the Maritime Alps. $\mathrm{He}$ is co-author of over 50 refereed journal publications. He currently leads radar research programs studying ocean surface winds, advanced radar methods for profiling the atmospheric boundary layer, and application of phased-array technology to meteorological radar polarimetry. His research interests include microwave imaging and interferometric techniques, radar oceanography, and radar meteorology.

Dr. Frasier is a senior member of the IEEE Geoscience and Remote Sensing Society, and a member of URSI Commission F, the American Geophysical Union, and the American Meteorological Society. From 2015 to 2018 he served as the Co-Chair of the Airborne Phased-Array Radar advisory panel to the National Center for Atmospheric Research. He served as an Associate Editor for the journal Radio Science from 2003 to 2010 and for the IEEE Transactions on Geoscience and Remote Sensing since 2010. 